(C) 2015 IEEE. Personal use of this material is permitted. Permission from IEEE must be obtained for all other uses, in any current or future media, including reprinting/republishing this material for advertising or promotional purposes, creating new collective works, for resale or redistribution to servers or lists, or reuse of any copyrighted component of this work in other works

\title{
Icing detection in unmanned aerial vehicles with longitudinal motion using an LPV unknown input observer
}

\author{
Damiano Rotondo, Andrea Cristofaro, Tor Arne Johansen, Fatiha Nejjari and Vicenç Puig
}

\begin{abstract}
This paper proposes a linear parameter varying (LPV) unknown input observer for the diagnosis of actuator faults and icing in unmanned aerial vehicles (UAVs). The accretion of ice layers on wings and control surfaces modifies the shape of the aircraft and alters the performance and controllability of the vehicles. The correct detection of this phenomenon is of paramount importance for the efficient implementation of de-icing techniques. The advantage of deriving the unknown input observer within the LPV framework is the possibility to deal with the nonlinearities of the UAV model by embedding them within some varying parameters. Results obtained with a Zagi Flying Wing simulator are used to validate the effectiveness of the proposed approach.
\end{abstract}

Keywords: Unknown input observers, linear parameter varying (LPV) systems, icing detection, unmanned aerial vehicles (UAVs).

\section{INTRODUCTION}

Unmanned aerial vehicles (UAVs) can be prone to the occurrence of icing when they are required to fly under critical and harsh conditions. The phenomenon of icing is a well recognized problem in aviation research since the early 1900s [1]. The formation of ice layers decreases the lift and, simultaneously, increases the drag and the mass of the vehicle, thus requiring additional engine power and implying a premature stall angle [2]. Inflight icing is typically caused by the impact of supercooled water droplets (SWD). When a water droplet is cooled, it does not freeze until it reaches very low temperatures; however, if the droplet impacts on the aircraft surface, it freezes immediately and ice accretes [3]. The rate and the severity of icing are determined by several factors, such as shape and roughness of the impacting surface, vehicle speed, air temperature and relative humidity [4].

This work has been supported by a grant from Iceland, Liechtenstein and Norway through the EEA Financial Mechanism. Operated by Universidad Complutense de Madrid (ref. 006-ABEL-IM-2014B). The authors also acknowledge support by the Spanish Ministry of Science and Technology through the projects CICYT ECOCIS (ref. DPI2013-48243-C21-R) and CICYT HARCRICS (ref. DPI2014-58104-R), by AGAUR through the contract FI-DGR 2014 (ref. 2014FI_B1_00172), by the DGR of the Generalitat de Catalunya (ref. 2014/SGR/374) and by the Research Council of Norway through the Centres of Excellence funding scheme (ref. 223254 - AMOS). A. Cristofaro is also supported by the ERCIM Alain Bensoussan Fellowship programme (ABCDE project - FP7 Marie Curie Actions).

D. Rotondo, F. Nejjari and V. Puig are with Advanced Control Systems Group (SAC), Universitat Politècnica de Catalunya (UPC), TR11, Rambla de Sant Nebridi, 10, 08222 Terrassa, Spain. V. Puig is also with Institut de Robotica i Informatica Industrial (IRI), UPC-CSIC, Carrer de Llorens i Artigas, 4-6, 08028 Barcelona, Spain. A. Cristofaro and T. A. Johansen are with Center for Autonomous Marine Operations and Systems (AMOS) and with Department of Engineering Cybernetics, Norwegian University of Science and Technology, Norway. e-mail: damiano(dot)rotondo(at)yahoo(dot)it
The consequences of icing are even more severe for small unmanned aircrafts due to their simple architecture and limited payload, this making them mostly unsuitable for the typical anti-icing and de-icing devices that are included in the standard equipments of large airplanes. Some advanced deicing systems for UAVs have been recently proposed based on carbon nanotechnology [5], [6]. However, due to power consumption, fault/icing detection schemes with fast and accurate responses are needed in order to guarantee the efficiency of these systems [7]. With the aim of decoupling icing effects from those of actuator faults, icing detection schemes based on unknown input observers [8], [9] and adaptive multiple-model approach [10] have been proposed. However, these icing diagnosis techniques have been designed using a linearized model of the aircraft, therefore they are reliable only as long as the linearized model is consistent with the nonlinear one.

In this regard, the novel contribution of this paper is to extend the unknown input observer setup proposed in [9] to the longitudinal nonlinear aircraft dynamics using linear parameter varying (LPV) methods. The LPV formulation allows to cope with nonlinear systems using standard control and estimation techniques that were developed for linear systems [11], [12]. Unlike linearization techniques, LPV methods do not involve any approximation, since they rely on an exact transformation of the original nonlinear system into a linear-like one, by incorporating all the original nonlinearities within some varying parameters that schedule the state space matrices [13] (the resulting model is referred to as quasi- $L P V$, due to the dependence of the varying parameters on endogenous signals). Hence, the proposed LPV unknown input observer-based icing detection scheme has the main advantage of being consistent with the aircraft dynamics for a wide range of operating conditions.

The paper is structured as follows. Section II presents the UAV model and shows how a quasi-LPV model can be obtained using a nonlinear embedding in the parameters approach. Also, the icing effect and wind turbulence models are presented. Section III is dedicated to the design of an LPV unknown input observer for the actuator fault and icing diagnosis. A case-study is used in Section IV to demonstrate the effectiveness of the proposed approach. Finally, Section $\mathrm{V}$ outlines the main conclusions and suggests some future work. 


\section{MODEL AND SETUP}

The longitudinal equations of motion of an aircraft, under low-angle-of-attack condition, are the following ${ }^{1}[14]$ :

$$
\begin{aligned}
& \dot{u}=-q w-g \sin \theta+\frac{\rho V_{a}^{2} S}{2 m}\left[-\left(C_{0}+C_{\alpha} \alpha\right) \cos \alpha\right. \\
& +\left(C_{L_{0}}+C_{L_{\alpha}} \alpha\right) \sin \alpha+C_{L_{q}} \sin \alpha-C_{D_{q}} \cos \alpha \quad c q \\
& +C_{L_{\bar{\delta}}} \sin \alpha-C_{D_{\bar{\delta}}} \cos \alpha \delta_{e}+\frac{\rho S_{\text {prop }} C_{\text {prop }}}{2 m} k_{m}^{2} \delta_{t}{ }^{2}-V_{a}^{2} \\
& \dot{w}=q u+g \cos \theta+\frac{\rho V_{a}^{2} S}{2 m}\left[-\left(C_{0}+C_{\alpha} \alpha\right) \sin \alpha\right. \\
& -\left(C_{L_{0}}+C_{L_{\alpha}} \alpha\right) \cos \alpha-C_{D_{q}} \sin \alpha+C_{L_{q}} \cos \alpha \frac{c q}{2 V a} \\
& \text { - } C_{D_{\overline{\bar{\varepsilon}_{S}}}} \sin \alpha+C_{L_{\bar{\delta}_{e}}} \cos \alpha \delta_{e} \quad \quad \\
& \dot{q}=\frac{\rho V_{a}^{2 e} S c}{2 J_{y}} C_{m_{0}}+C_{m_{a}} \alpha+C_{m_{q}} \underset{z V_{a}}{c q}+C_{m_{\delta_{e}}} \delta_{e} \\
& \dot{\theta}=q
\end{aligned}
$$

where $u$ and $w$ are the aircraft horizontal and vertical velocity relative to the wind, in the vehicle body coordinate system, $q$ is the pitch rate and $\theta$ is the pitch angle, $g$ is the gravitational acceleration, $\rho$ is the air density, $S$ is the wing surface area, $m$ is the airframe mass, $\alpha$ is the angle-ofattack, $c$ is the mean aerodynamic chord of the wing, $S_{\text {prop }}$ is the area of the propeller, $k_{m}$ is the constant that specifies the efficiency of the motor, $J_{y}$ is an element of the inertia matrix and $V_{a}$ is the airspeed with respect to the air mass. The inputs entering the system are the throttle deflection $\delta_{t}$ and the elevator efficiency $\delta_{e}$. Finally, the non-dimensional coefficients $C_{i}$ are usually referred to as stability and control derivatives.

Taking into account the relations between $u, w, V_{a}$ and $\alpha$ :

$$
V_{a}={ }^{\prime} \vec{u}^{2}+w^{2} \quad \alpha=\arctan { }_{u}
$$

the nonlinear model (1)-(4) can be brought to a quasiLPV form using the nonlinear embedding in the parameters approach [15], [16], as follows:

$$
\dot{x}=A(u, w, q, \theta) x+B(u, w) \mathrm{U}+d(\theta)
$$

where $x=(u, w, q, \theta)^{T}$ is the state vector, $\mathrm{u}=\left(\delta_{t}^{2}, \delta_{e}\right)^{T}$ is the input vector, and the matrices $A(u, w, q, \theta), B(u, w), d(\theta)$ are given by:

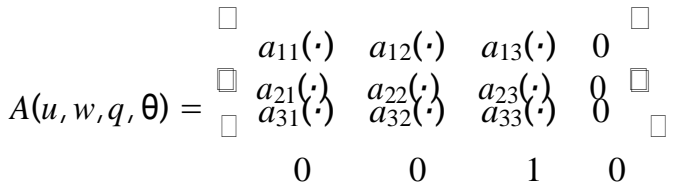

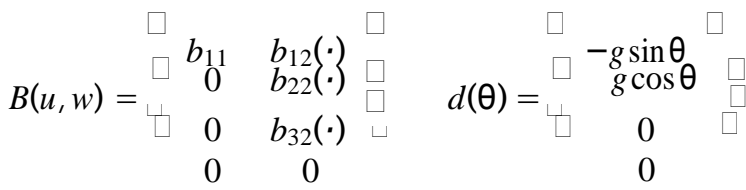

\footnotetext{
${ }^{1}$ To ease the notation, the dependence of variables on time $t$ is omitted.
}

$$
\begin{aligned}
& \text { where: }{ }_{\rho u S}{ }^{1} \quad \underline{S \text { prop }}_{\text {prop }}{ }^{\text {I }} \\
& a_{11}(\cdot)={ }_{2 m} \quad C_{L_{0}}+C_{L_{\alpha}} \alpha \sin \alpha-C_{D_{0}}+C_{D_{\alpha}} \alpha \cos \alpha-\quad S
\end{aligned}
$$

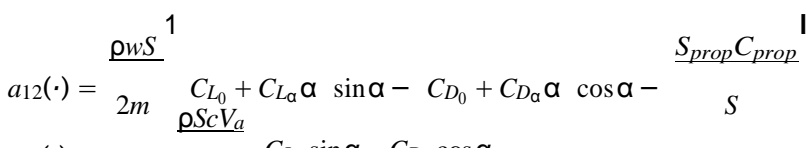

$$
\begin{aligned}
& a_{13}(\cdot)=-w+\quad C_{L_{q}} \sin \alpha-C_{D_{q}} \cos \alpha \\
& \begin{array}{l}
a_{13}(\cdot)=-w+ \\
a()=-\left.\underline{\rho u S}\right|^{4 m}
\end{array} \\
& { }_{21} \cdot-\frac{2 m}{2 m}, C_{D_{0}}+C_{D_{\alpha}} \alpha \sin \alpha+C_{L_{0}}+C_{L_{\alpha}} \alpha \cos \alpha \\
& a_{22}(\cdot)=-\underline{\rho w S} C_{D}+C_{D_{\alpha}} \alpha \sin \alpha+C_{L} \quad L_{\alpha}
\end{aligned}
$$

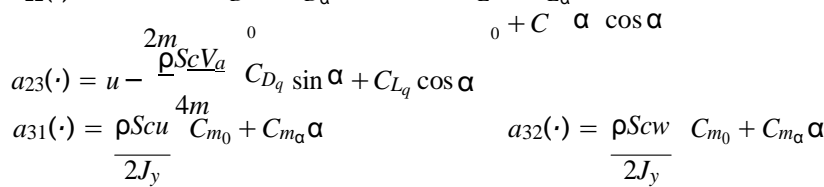

$$
\begin{aligned}
& a_{33}(\cdot)=\frac{\rho V_{a} S c^{2}}{4 J_{y}} C_{m_{q}} \quad b_{11}=\frac{\rho S_{\text {prop }} C_{\text {prop }}}{2 m} k^{2} \\
& b_{12}(\cdot)=\rho S v^{2}\left(C_{L} \quad D\right. \\
& \overline{\rho V}^{2 m} S\left({ }^{\delta_{e}} \sin \alpha-C_{\delta_{e}} \cos \alpha\right. \\
& b_{22}(\cdot)=-\frac{a}{2 m} C_{D_{\bar{\delta}_{e}}} \sin \alpha+C_{L_{\bar{\delta}}} \cos \alpha \quad b_{32}(\cdot)=\frac{\rho V^{2} S c}{2 J_{y}} C_{m_{\bar{\delta}_{e}}}
\end{aligned}
$$

The wind effect can be modeled as an additive disturbance vector $\mathcal{W}$ given by:

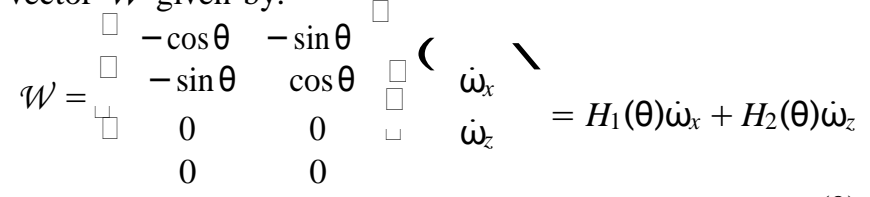

where $\dot{\omega}_{x}$ and $\dot{w}_{z}$ are the wind accelerations in the horizontal and vertical directions in the inertial frame, respectively. Assuming that the UAV is equipped with airspeed measurement device (pitot tube), GPS and inertial sensors, all state variables are supposed to be available and hence the output matrix of the system verifies $C=I_{4 \times 4}$.

Actuator faults may affect the system and these can be represented as an unknown input term $F$ given by:

$$
F=\begin{array}{cc}
b_{11} & b_{12}(\cdot) \\
0 & b_{22}(\cdot) \\
0 & b_{32}(\cdot) \\
0 & 0
\end{array} C^{\square} \boldsymbol{\Phi}_{t}=B_{1} \phi_{t}+B_{2}(u, w) \phi_{e}
$$

where $\phi_{t}$ and $\phi_{e}$ correspond to faults in propellers/engines and elevator, respectively.

\section{A. Icing effect model}

The accretion of clear ice on the aircraft surfaces modifies the stability and control derivatives according to the following linear model [17]:

$$
C_{i}^{\mathrm{k}}=\left(1+\eta K_{i}\right) C_{i}
$$

where $\eta$ is the icing severity factor and the coefficient $K_{i}$ depends on aircraft design and atmospheric conditions. The clean condition corresponds to $\eta=0$, while the worst icing condition occurs for $\eta=0.2$.

As a consequence, the overall icing effect can be modeled as an additive time-dependent disturbance term $\mathcal{E}(u, w, q) \eta$, where $\eta$ is a scalar unknown quantity and the vector $\mathcal{E}(u, w, q)$ is given by:

$$
\mathcal{E}(u, w, q)=\mathcal{E}_{1}(u, w, q) \quad \mathcal{E}_{2}(u, w, q) \quad \mathcal{E}_{3}(u, w, q) \quad 0^{T}
$$


with:

$$
\begin{aligned}
& \mathcal{E}_{1}(u, w, q)=\stackrel{\rho V^{2} S}{\underline{a}}\left[\left(K_{L} C_{L}+K_{L} C_{L} \alpha\right) \sin \alpha\right. \\
& \left.\stackrel{2 m}{2 m} \stackrel{0}{0} \quad{ }^{0} K_{D_{0}} C_{D_{0}}+K_{D_{\alpha}} C_{D_{\alpha}} \alpha\right) \cos \alpha \\
& +K_{L_{q}} C_{L_{q}} \sin \alpha-K_{D_{q}} C_{D_{q}} \cos \alpha \frac{c q}{2 V a} \\
& +K_{L_{\delta_{e}}} C_{L_{\tilde{L}_{e}}} \sin \alpha-K_{D_{\delta_{e}}} C_{D_{\delta_{e}}} \cos \alpha \delta_{e} \\
& \mathcal{E}_{2}(u, w, q)=-\frac{\rho V_{a}^{2} S}{2 m}\left[\left(K_{D_{0}} C_{D_{0}}+K_{D_{\alpha}} C_{D} \alpha\right) \sin \alpha\right. \\
& +\left(K_{L_{0}} C_{L_{0}}+K_{L_{\alpha}} C_{L_{\alpha}} \alpha\right) \cos \alpha \\
& +K_{D_{q}} C_{D_{q}} \sin \alpha+K_{L_{q}} C_{L_{q}} \cos \alpha \frac{c q}{2 V^{a}} \\
& +K_{D_{\bar{\delta}}} C_{D_{\bar{\delta}_{e}}} \sin \alpha+K_{L_{\delta_{e}}} C_{L_{\bar{\delta}_{e}}} \cos \alpha \bar{\delta}_{e} \\
& \mathcal{E}_{3}(u, w, q)=\frac{\rho V^{2}{ }^{2} S c}{2 J_{y}}\left(K_{m} C_{0} C_{m}+K_{m} C_{m} \alpha\right. \\
& +K_{m_{q}} C_{m_{q}} \frac{c q}{2 V^{a}}+K_{m_{\delta_{e}}} C_{m_{\delta_{e}}} \delta_{e}
\end{aligned}
$$

\section{B. Wind turbulence model}

In order to model in a realistic way the wind gusts, the widely accepted Dryden wind turbulence model is used [18]. The Dryden model uses spatially varying stochastic processes to represent the components of the gusts, specifying their power spectral density.

\section{ACtUATOR FAULTS AND ICING DIAGNOSIS}

Taking into account the wind, the actuator faults and the icing, the quasi-LPV equation (6) is slightly modified, as follows:

$$
\dot{x}=A(u, w, q, \theta) x+B(u, w) \mathrm{U}+B_{u n}(u, w, q, \theta) \mathrm{u}_{u n}+d(\theta)
$$

where $U_{u n}=\left(\dot{\omega}_{x}, \dot{\omega}_{z}, \phi_{t}, \phi_{e}, \eta\right)^{T}$ is the unknown input vector, and the matrix $B_{u n}(u, w, q, \theta)$ is given by:

$$
B_{u n}(u, w, q, \theta)=\left(\begin{array}{lllll}
H_{1}(\theta) & H_{2}(\theta) & B_{1} & B_{2}(u, w) & E(u, w, q)
\end{array}\right)
$$

For further reasoning, let us rewrite (17) in a more general form:

$$
\dot{x}=A(\vartheta) x+B(\vartheta) \mathrm{u}+B_{u n}(\vartheta) \mathrm{U}_{u n}+d(\vartheta)
$$

where $\vartheta$ is some varying parameter vector, containing exogenous variables, endogenous variables (e.g. states and/or inputs), or a combination of them.

In the following, an LPV unknown input observer for (18) is proposed. As remarked by [19], the main advantage of such observer is that, if some structural conditions are met, the parameters can be designed such that the resulting estimation error is independent of some inputs of the system, even if these are not measured directly.

Theorem 1: Let $R(\vartheta)$ and $F(\vartheta)$ be some given matrix functions, and calculate the following matrix functions:

$$
\begin{aligned}
T(\vartheta) & =I-R(\vartheta) \\
S_{1}(\vartheta) & =R(\vartheta) A(\vartheta)-F(\vartheta) \\
S_{2}(\vartheta) & =F(\vartheta) T(\vartheta)
\end{aligned}
$$

Then:

$\dot{z}=F(\vartheta) z+R(\vartheta) B(\vartheta) \mathrm{U}+S(\vartheta) x-T(\vartheta) x+d(\vartheta)-T(\vartheta) d(\vartheta)$

$\hat{x}=z+T(\vartheta) x$

where $\dot{T}(\vartheta)$ is the time derivative of $T(\vartheta)$ and:

$$
S(\vartheta)=S_{1}(\vartheta)+S_{2}(\vartheta)
$$

is an unknown input observer for (18), with the estimation error $\varepsilon=x-\hat{x}$ given by:

$$
\dot{\varepsilon}=F(\vartheta) \varepsilon+R(\vartheta) B_{\text {un }}(\vartheta) \mathrm{U}_{\text {un }}
$$

Proof: The estimation error, taking into account (18) and

(22)-(23), is given by:

$$
\begin{aligned}
\dot{\varepsilon} & =[A(\vartheta)-T(\vartheta) A(\vartheta)-S(\vartheta)] x-F(\vartheta) z \\
& +(I-R(\vartheta)-T(\vartheta)) B(\vartheta) \mathrm{U}+[I-T(\vartheta)] B_{\text {un }}(\vartheta) \mathrm{U}_{\text {un }}
\end{aligned}
$$

Taking into account (19), (26) can be rewritten as:

$$
\dot{\varepsilon}=[R(\vartheta) A(\vartheta)-S(\vartheta)]_{x}-F(\vartheta) z+R(\vartheta) B_{u n}(\vartheta) \mathrm{U}_{u n}
$$

Then, combining (20)-(21) with (24), it can be shown that (27) is equivalent to:

$$
\dot{\varepsilon}=[F(\vartheta)-F(\vartheta) T(\vartheta)] x-F(\vartheta) z+R(\vartheta) B_{u n}(\vartheta) \mathrm{U}_{u n}
$$

It is easy to see that (25) results from (28) taking into account (23), which completes the proof. D

Notice that $F(\vartheta)$ can be chosen as a constant matrix $F$ through an appropriate choice of the matrix $S_{1}(\vartheta)$, which allows to assign some desired eigenvalues of $F$ and assure convergence to zero of the estimation error $\varepsilon$ when $\mathrm{U}_{u n}=$ 0 . On the other hand, the matrix $R(\vartheta)$ can be chosen to constrain the range of the matrix $R(\vartheta) B_{u n}(\vartheta)$, in such a way that different output directions of the residuals are assigned for wind disturbance, actuator faults and icing effects, respectively, with the aim of identifying the cause for some detected system malfunctions.

Due to the superposition of effects and the lack of degrees of freedom in the unknown input observer design, it is not possible to decouple completely the wind disturbance and icing effects from the actuator faults. However, it is still possible to design the unknown input observer matrices such that a successful fault/icing diagnosis can be achieved.

To this purpose, let us notice that as long as $\cos \theta /=0$, the following condition holds:

$\mathcal{E}(u, w, q, \theta)=\mathcal{E}(t) \in \operatorname{span}^{\prime} \quad B_{1} \quad B_{2}(u, w) \quad H_{2}(\theta) \quad \forall t \geq 0$

Let us denote by $e_{1}, e_{2}, e_{3}, e_{4}$ the canonical basis vectors of the output space $\mathrm{R}^{4}$. Referring to the system (16), the target is to design the unknown input observer matrices with the following properties:

$$
\begin{aligned}
& R(u, w, q, \theta) B_{1}=b_{11} e_{1} \\
& R(u, w, q, \theta) B_{2}(u, w)=V_{a}^{2} e_{2} \\
& R(u, w, q, \theta) H_{2}(\theta)=\cos \theta e_{3} \\
& F e_{i}=\lambda_{i}^{F} e_{i} \quad \forall i=1,2,3,4
\end{aligned}
$$


where $\lambda_{i}^{F}, i=1,2,3,4$, are the desired eigenvalues of the matrix $F\left(\lambda_{i}^{F} \in \mathrm{C}^{-}\right.$in order to assure the stability of the observer). (i2):

It is easy to check that the following matrix satisfies (30)-

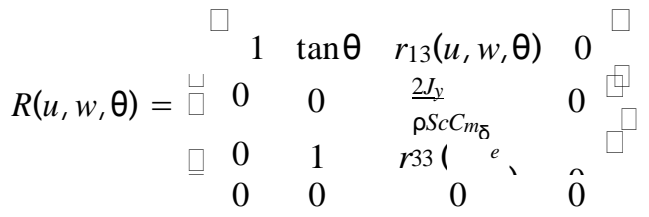

$$
\begin{aligned}
& r_{13}(u, w, \theta)=\frac{J_{y}}{m c C^{m_{\delta_{e}}}} r\left(C_{D_{\bar{\delta}_{e}}}+C_{L_{\bar{\delta}_{e}}} \tan \theta \cos \alpha\right. \\
& +C_{D_{\bar{\delta}_{e}}} \tan \theta-C_{L_{\bar{\delta}_{e}}} \sin \boldsymbol{\alpha}^{\mathbf{I}} \\
& r_{33}(u, w)=\frac{J_{y}}{m c C_{m_{\sigma_{e}}}} C_{L_{\bar{\delta}_{e}}} \cos \alpha+C_{D_{\bar{\delta}_{e}}} \sin \alpha
\end{aligned}
$$

Since the state of the system is completely known, $z(0)$ can be chosen as $z(0)=\left(I-T_{0}\right) x(0)$, where $T_{0}$ denotes the initial value of the matrix $T(u, w, q, \theta)$, such that from (23) results $\hat{x}(0)=x(0)$, i.e. $\varepsilon(0)=0$. As a consequence, the estimation error belongs to a linear subspace that is determined by the range of the matrix:

$$
R(u, w, q, \theta)^{\prime} B_{1} \quad B_{2}(u, w) \quad H_{2}(\theta)
$$

It has been noticed that the frequency content of the variables $\varepsilon_{i}(t), i=1,2,3$, changes under faults/icing occurrence. In fact, in absence of faults/icing, the residuals are excited only by the wind acceleration, which is mostly a high frequency disturbance. On the other hand, the actuator faults and icing effects increase the low frequency content of the variables $\varepsilon_{i}(t)$. Thus, the introduction of low-frequency residuals $\varphi_{1}(t), \varphi_{2}(t), \varphi_{3}(t)$ provides further information about the presence of faults/icing. More specifically, the lowfrequency residuals are defined as follows:

$$
\psi_{i}(t)=\begin{array}{ll}
\text { not defined } & \text { if } t<T \\
20 \log \left|\Phi_{i}(t, T)\right| & \text { if } t \geq T
\end{array}
$$

with $\Phi_{i}(t, T)=F_{0}\left\{\varepsilon_{i}[t-T, t]\right\}$, where $F_{0}$ denotes the Fourier transform evaluated at the frequency $f=0 \mathrm{~Hz}$, and $\varepsilon_{i}[t-T, t]$ is the sequence extracted from $\varepsilon_{i}(t)$ in the time interval $[t-T, t]$. The parameter $T$ should be chosen taking into account the tradeoff between assuring a fast detection of faults/icing occurrence (smaller $T$ ) and highlighting the faults/icing effects with respect to the wind disturbance ones (bigger $T$ ).

Then, the following algorithm is proposed to decide about the occurrence of faults/icing.

\section{Decision Algorithm.}

\begin{tabular}{|c|c|c|c|c|c|}
\hline Param. & Value & Param. & Value & Param. & Value \\
\hline$m$ & $1.56 \mathrm{~kg}$ & $C_{L_{0}}$ & 0.09167 & $C_{D_{q}}$ & 0 \\
\hline$J_{y}$ & $0.0576 \mathrm{kgm}^{<}$ & $C_{D_{0}}$ & 0.01631 & $C_{m}$ & -1.3990 \\
\hline$S$ & $0.2589 \mathrm{~m}^{2}$ & $C_{m_{0}}^{0}$ & -0.02338 & $C_{L_{\bar{\delta}}}^{q}$ & 0.2724 \\
\hline c & 0.3302 & $n \quad C_{L_{\alpha}}$ & 3.5016 & $C_{D_{\bar{\delta} e}}$ & 0.3045 \\
\hline$S_{p r o p}$ & $\begin{array}{c}0.0314 \mathrm{~m}^{2} \\
1.2682 \mathrm{~kg} / \mathrm{m}^{3}\end{array}$ & ${\stackrel{C}{D_{\alpha}}}_{m_{\alpha}}$ & $\begin{array}{r}0.2108 \\
-0.5675\end{array}$ & ${\stackrel{C}{m_{\delta_{e}}}}_{\text {prop }}^{C_{e}}$ & $\begin{array}{c}-0.3254 \\
1.0\end{array}$ \\
\hline$k_{m}$ & 20 & $C_{L_{q}}$ & 2.8932 & & \\
\hline
\end{tabular}

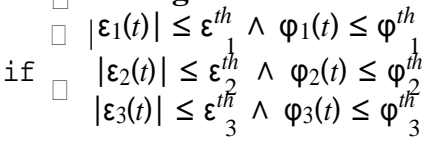

$$
\begin{aligned}
& \text { then "no faults/no icing" } \\
& \left|\varepsilon_{1}(t)\right|>\varepsilon^{\text {th }} \vee \varphi_{1}(t)>\varphi^{\text {th }} \\
& \text { if }\left|\varepsilon_{2}(t)\right| \leq \varepsilon_{2}^{t^{t h}} \wedge \varphi_{2}(t) \leq \varphi_{12}^{\text {th }} \\
& \left|\varepsilon_{3}(t)\right| \leq \varepsilon_{3}^{t^{2}} \wedge \varphi_{3}(t) \leq \varphi^{t t^{2}}
\end{aligned}
$$

TABLE I

SYSTEM PARAMETERS VALUES

$$
\begin{aligned}
& \text { then "fault in thrust"' } \\
& \text { if }\left|\varepsilon_{1}(t)\right| \leq \varepsilon^{\text {th }} \wedge \varphi_{1}(t) \leq \varphi_{\text {th }}^{\text {th }} \\
& { }_{3} \wedge \varphi_{3}(t) \leq \varphi_{3} \\
& \text { then "fault in elevator" } \\
& \text { else "'possible icing" }
\end{aligned}
$$

$$
\text { th th th th th th }
$$

Remark 1: The thresholds $\varepsilon_{1}, \varepsilon_{2}, \varepsilon_{3}, \varphi_{1}, \varphi_{2}, \varphi_{3}$ should be calculated in such a way that the residuals never exceed them due to the wind turbulence. Also notice that the component $\dot{\omega}_{z}$ will only affect the residuals $\varepsilon_{3}(t)$ and $\varphi_{3}(t)$ due to (32).

Remark 2: Taking into account (19), $\dot{T}(\vartheta)=-\dot{R}(\vartheta)$. Due to the presence of $\cos \alpha$ and $\sin \alpha$ in $r_{13}(u, w, \theta)$ and $r_{33}(u, w, \theta)$, the corresponding elements of the matrix $\dot{T}$ will contain $\dot{\alpha}$, which is affected by the unknown faults and icing terms. As a consequence, the decoupling achieved by the unknown input observer designed using Theorem 1 is not perfect. However, it has been noticed that these elements are of small size, and can be neglected without invalidating the effectiveness of the proposed approach. In particular, $\dot{T}(\vartheta)$ is approximated with the following matrix, obtained from (34) assuming a constant $\alpha$ :

$$
\begin{aligned}
& \dot{T}(u, w, q, \theta)=\begin{array}{cccl}
\sqcup & \dot{i}_{12}(q, \theta) & \tilde{t}_{13}(u, w, q, \theta) & 0 \\
-u & 0 & 0 & 0 \\
\square & 0 & 0 & 0 \\
u & 0 & 0 & 0
\end{array}
\end{aligned}
$$

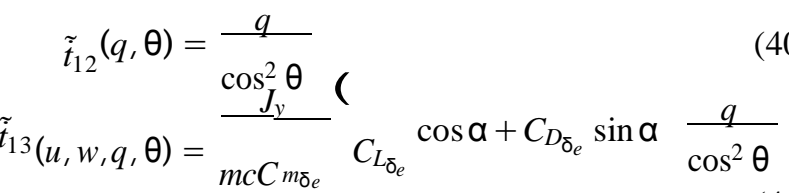

\section{CASE STUDY}

Let us consider the case study of a small unmanned aircraft, the Zagi Flying Wing. The parameters appearing in the nonlinear longitudinal model (1)-(4) are provided in Table I.

The aircraft is subject to wind accelerations calculated using the Dryden wind turbulence model provided by the Aerospace Toolbox of MATLAB, for an aircraft flying at a height $h=150 \mathrm{~m}$ and with a light probability of exceedance of high-altitufe intensity $y_{\text {is }}$ supposed to be controlled by an autopilot, responsible to maintain the horizontal velocity 
TABLE II

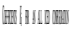

\begin{tabular}{cccccc}
\hline Coeff. & Value & Coeff. & Value & Coeff. & Value \\
\hline$K_{L_{0}}$ & 0 & $K_{L_{\alpha}}$ & -0.5000 & $K_{L_{q}}$ & -0.0675 \\
$K_{L_{\bar{\delta}}}$ & -0.4770 & $K_{D_{0}}$ & 2.5610 & $K_{D_{\alpha}}$ & 0 \\
$K_{D_{q}}$ & 0 & $K_{D_{\bar{\delta}_{e}}}$ & 0 & $K_{m_{0}}$ & 0 \\
$K_{m_{\alpha}}$ & -0.4960 & $K_{m_{q}}$ & -0.1755 & $K_{m_{\bar{\delta}}}$ & -0.5000 \\
\hline
\end{tabular}

around a desired value $u_{r e f}=20 \mathrm{~m} / \mathrm{s}$ with the following reference pitch angle:

$$
\theta_{\text {ref }}=\begin{array}{cc}
0 & t \leq 100 s \\
\frac{(t-100)}{1500} & 100 s \leq t \leq 250 s \\
\frac{(450-t)}{2000} & 250 s \leq t \leq 450 s \\
0 & t>450 s
\end{array}
$$

Three different fault scenarios have been analyzed, namely:

1) Fault in thrust

2) Fault in elevator

3) Icing

\section{A. Fault scenario 1 (FS1)}

The effective thrust input $\delta_{t}$ is subject to a loss of efficiency with respect to its nominal value $\delta_{t}^{*}$. The fault is linearly incipient, such that the loss of efficiency starts at time $t=200 \mathrm{~s}$ and equals $\delta_{t}(t)=0.7 \delta_{t}^{*}(t)$ starting from time $t=210 s$.

\section{B. Fault scenario 2 (FS2)}

The effective elevator deflection $\delta_{e}$ is subject to a loss of efficiency with respect to its nominal value $\delta_{e}^{*}$. The fault is linearly incipient, such that the loss of efficiency starts at time $t=200 \mathrm{~s}$ and equals $\bar{\delta}_{e}(t)=0.9 \delta_{e}^{*}(t)$ starting from time $t=210 s$.

\section{Fault scenario 3 (FS3)}

The aircraft is subject to icing, i.e. the stability and control derivatives are modified according to (11), taking into account the coefficients $K_{i}$ listed in Table $\mathrm{II}^{2}$. The icing starts at time $t=200 \mathrm{~s}$ and slowly increases $\eta$ from 0 to 0.2 , such that $\eta=0.2$ starting from time $t=400 \mathrm{~s}$.

Figs. 1-3 show the evolution of the temporal residuals $\varepsilon_{1}(t), \varepsilon_{2}(t)$ and $\varepsilon_{3}(t)$ in the considered fault scenarios. It can be seen that in fault scenario 1 (fault in the thrust), the only residual that differs considerably from the normal behavior (before $t=200 \mathrm{~s}$ ) is $\varepsilon_{1}(t)$. Looking at $\varepsilon_{2}(t)$ it can be seen that both the fault in elevator (fault scenario 2) and the icing (fault scenario 3) act on this residual. However, since the temporal residual $\varepsilon_{3}(t)$ is not excited enough to overcome the effect of the wind acceleration disturbance, a successful fault isolation

\footnotetext{
${ }^{2}$ The coefficients $K_{i}$ used in this work have been computed mimicking the proportional variation of the stability and control derivatives for a Twin Otter aircraft subject to all iced condition [17], and they could differ in the case of a real Zagi Flying Wing UAV. However, since the proposed LPV unknown input observer does not depend on the values of these coefficients, it can be expected that similar results would be obtained with different values of the coefficients $K_{i}$.
}

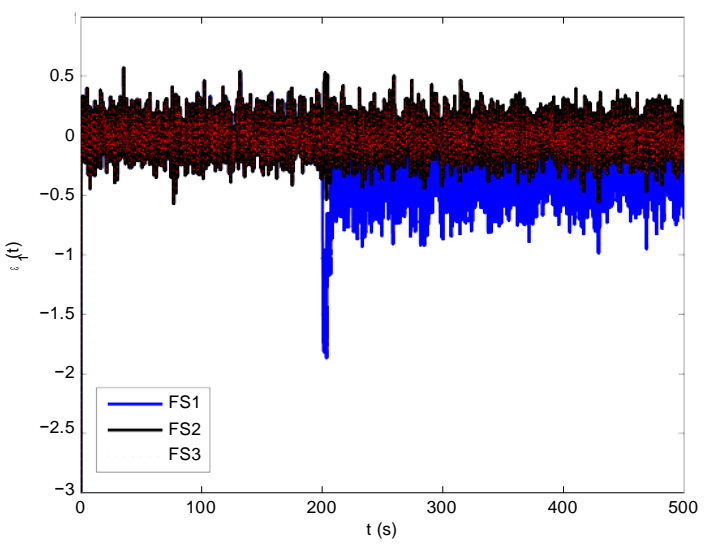

Fig. 1. Temporal residual $\varepsilon_{1}(t)$.

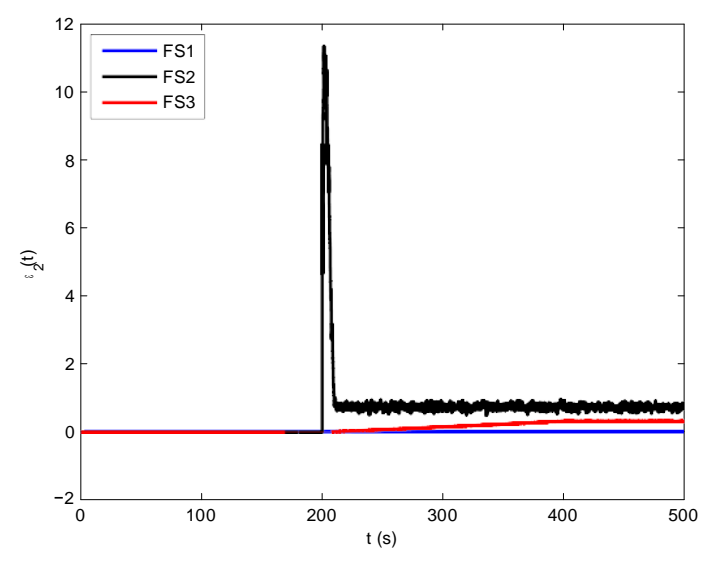

Fig. 2. Temporal residual $\varepsilon_{2}(t)$.

using only information coming from the temporal residuals cannot be achieved.

However, the Decision Algorithm proposed in the previous section also exploits the information coming from the lowfrequency residuals $\varphi_{1}(t), \varphi_{2}(t)$ and $\varphi_{3}(t)$, obtained with $T=40 \mathrm{~s}$, and depicted in Figs. 4-6. In particular, by looking at $\varphi_{3}(t)$, it can be seen that the icing affects strongly this residual, unlike the elevator fault, such that a discrimination between fault in elevator and icing is possible.

\section{CONCLUSiOnS}

This paper has proposed a method for icing detection in UAVs using an LPV unknown input observer. The decision algorithm identifies correctly unexpected effects in the system dynamics due to actuator faults and icing, using information coming from temporal and low-frequency residuals. The case study of a Zagi Flying Wing UAV has allowed to validate the effectiveness of the proposed approach.

In this work, only the longitudinal motion of the aircraft has been considered. Future investigation will focus on the extension of the results to the complete 9-DOF aircraft model and the inclusion of uncertainties in the model and noise in the sensors. 


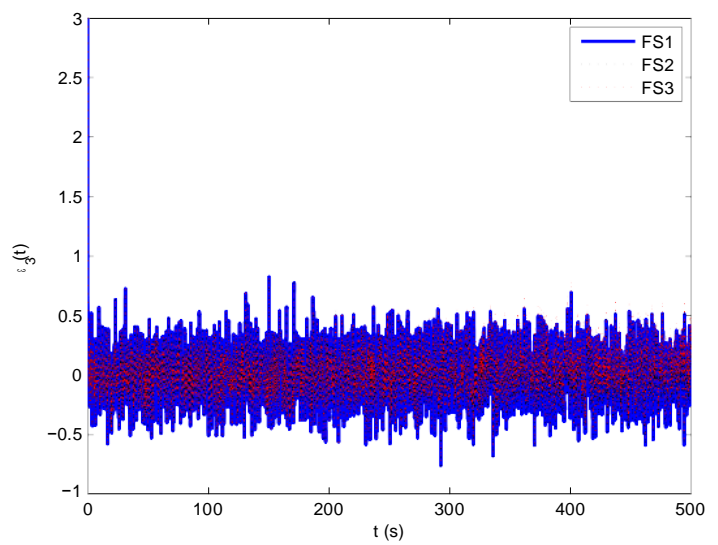

Fig. 3. Temporal residual $\varepsilon_{3}(t)$.

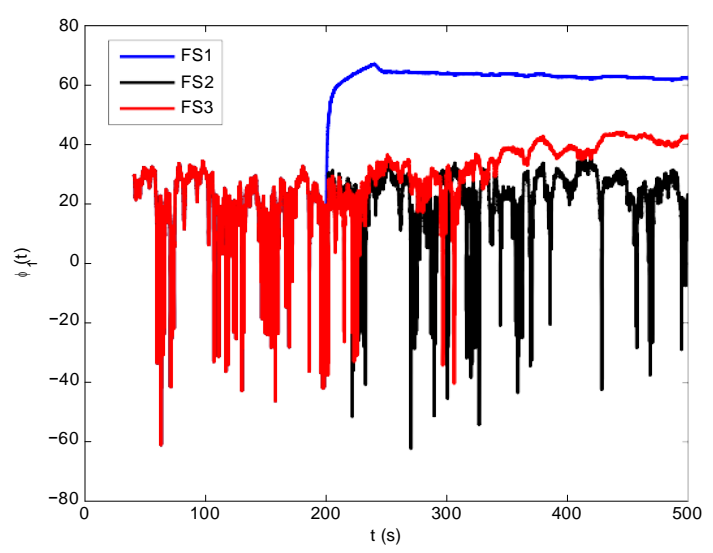

Fig. 4. Low-frequency residual $\varphi_{1}(t)$.

\section{REFERENCES}

[1] F. Caliskan and C. Hajiyev, "A review of in-flight detection and identification of aircraft icing and reconfigurable control," Progress in Aerospace Sciences, vol. 60, pp. 12-34, 2013.

[2] R. W. Gent, N. P. Dart, and J. T. Cansdale, “Aircraft icing," Phil. Trans. of the Royal Soc. of London. Series A: Mathematical, Physical and Engineering Sciences, vol. 358, pp. 2873-2911, 2000.

[3] T. G. Myers and D. W. Hammond, "Ice and water film growth from incoming supercooled droplets," Int. Journal of Heat and Mass Transfer, vol. 42, pp. 2233-2242, 1999.

[4] M. B. Bragg, A. P. Broeren, and L. Blumenthal, "Iced-airfoil aerodynamics,” Progress in Aerospace Sciences, pp. 323-362, 2005.

[5] S. Bone and M. Duff, "Carbon nanotubes to de-ice UAVs," http://136.142.82.187/eng12/Author/data/2122.docx, Technical report, 2012.

[6] K. L. Sørensen, A. S. Helland, and T. A. Johansen, "Carbon nanomaterial-based wing temperature control system for in-flight antiicing and de-icing of unmanned aerial vehicles,” IEEE Aerospace Conference, 2015.

[7] C. Hajiyev and F. Caliskan, Fault diagnosis and reconfiguration in flight control systems. Springer Science \& Business Media, 2003.

[8] M. Tousi and K. Khorasani, "Robust observer-based fault diagnosis for an unmanned aerial vehicle,” in Systems Conference (SysCon), 2011 IEEE International, 2011, pp. 428-434.

[9] A. Cristofaro and T. A. Johansen, “An unknown input observer approach to icing detection for unmanned aerial vehicles with linearized longitudinal motion,” American Control Conference, 2015.

[10] $\_$, "Icing detection and identification for unmanned aerial vehicles:

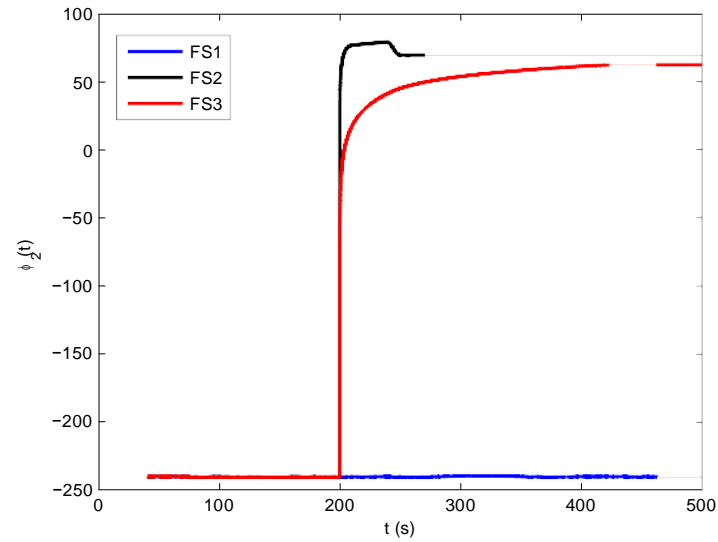

Fig. 5. Low-frequency residual $\varphi_{2}(t)$.

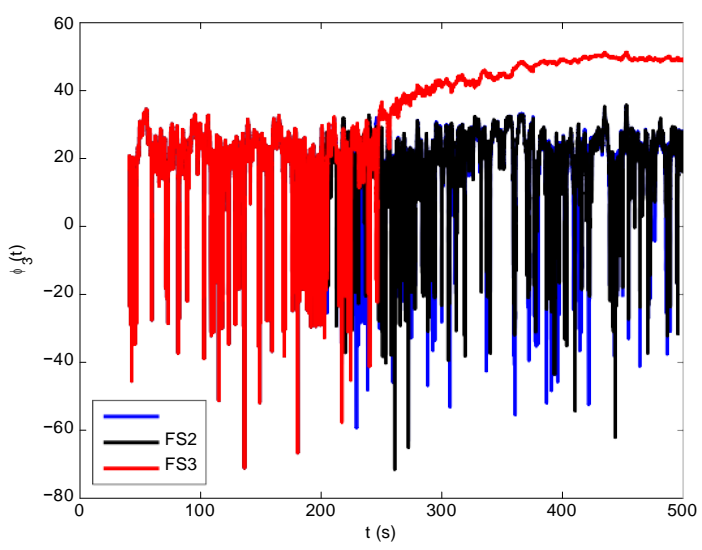

Fig. 6. Low-frequency residual $\varphi_{3}(t)$.

multiple model adaptive estimation,” European Control Conference, 2015.

[11] D. Rotondo, F. Nejjari, and V. Puig, "A virtual actuator and sensor approach for fault tolerant control of LPV systems,” Journal of Process Control, vol. 24, no. 3, pp. 203-222, 2014.

[12] D. Rotondo, F. Nejjari, V. Puig, and J. Blesa, "Model reference FTC for LPV systems using virtual actuator and set-membership fault estimation," International Journal of Robust and Nonlinear control, vol. 25, no. 5, pp. 735-760, 2015.

[13] J. S. Shamma, "An overview of LPV systems," in Control of Linear Parameter Varying Systems with Applications, J. Mohammadpour and C. Scherer, Eds. Springer, 2012, pp. 3-26.

[14] R. W. Beard and T. W. McLain, Small Unmanned Aircraft: Theory and Practice. Princeton, NJ, USA: Princeton University Press, 2012.

[15] A. Kwiatkowski, M.-T. Boll, and H. Werner, “Automated generation and assessment of affine LPV models," in Proceedings of the 45th IEEE Conference on Decision and Control, 2006, pp. 6690-6695.

[16] D. Rotondo, V. Puig, F. Nejjari, and M. Witczak, "Automated generation and comparison of Takagi-Sugeno and polytopic quasi-LPV models,” Fuzzy Sets and Systems, vol. in press, 2015.

[17] M. B. Bragg, T. Hutchinson, J. Merret, R. Oltman, and D. Pokhariyal, "Effect of ice accretion on aircraft flight dynamics," in Proceedings of the 38th AIAA Aerospace Science Meeting and Exhibit, 2000.

[18] F. M. Hoblit, Gust loads on aircraft: concept and applications. Washington, DC, USA: American Institute of Aeronautics and Astronautics, 1988.

[19] J. Chen, R. Patton, and H. Zhang, "Design of unknown input observers and robust detection filters,” International Journal of Control, vol. 63, pp. 85-105, 1996. 